\title{
Análisis jurídico del ascenso y cese de las personas adultas mayores en el servicio público
}

\section{Legal analysis of the promotion and cessation of older adults in public service}

\author{
Yaneth Lucia Valverde-Torres \\ us.yanhetvalverde@uniandes.edu.ec \\ Universidad Regional Autónoma de los Andes, Santo Domingo \\ Ecuador \\ https://orcid.org/0000-0002-0891-286X \\ Sonia Elizabeth Barreno-Zambrano \\ ds.soniaebz33@uniandes.edu.ec \\ Universidad Regional Autónoma de los Andes, Santo Domingo \\ Ecuador \\ https://orcid.org/0000-0001-9481-8652
}

Recepción: 15 de septiembre 2021

Revisado: 25 octubre 2021

Aprobación: 15 de noviembre 2021

Publicación: 01 de diciembre 2021 


\title{
RESUMEN
}

El trabajo de investigación, se planteó como objetivo general analizar jurídica y doctrinariamente la discriminación por edad que sufren las personas adultas mayores para el ascenso y permanencia en el servicio público, en Ecuador. Desde el paradigma cualitativo, se examinaron los contenidos de fondo del análisis documental y bibliográfico, legal; mediante los métodos analítico-sintético, inductivo-deductivo. Se realizaron entrevistas y recogió información mediante ficha bibliográfica. Como resultado se obtuvo que la edad no es una limitante para el ascenso a un puesto de mayor jerarquía y muy por el contrario se debería considerar a los servidores públicos de larga trayectoria, que hayan cumplido los 65 años de edad al beneficio de las instituciones pública. Se concluye interponer la garantía jurisdiccional de acción de inconstitucionalidad al artículo 81 de la Ley Orgánica de Servicio Público, la cual puede ser presentada por cualquier persona o grupo de personas, en calidad de funcionario público(s).

Descriptores: Administración pública; vejez; trabajo; justicia social; garantías jurídicas. (Palabras tomadas de Tesauro UNESCO).

\begin{abstract}
The research work was proposed as a general objective to analyze legally and doctrinally the age discrimination suffered by older adults for promotion and permanence in public service, in Ecuador. From the qualitative paradigm, the substantive contents of the documentary and bibliographic analysis, legal; through analytical-synthetic, inductive-deductive methods. Interviews were conducted and information was collected through a bibliographic file. As a result, it was obtained that age is not a limitation for promotion to a higher-ranking position and, on the contrary, long-standing public servants who have reached 65 years of age should be considered for the benefit of public institutions. It is concluded to bring the jurisdictional guarantee of unconstitutionality action to article 81 of the Organic Law of Public Service, which can be presented by any person or group of people, as public official (s).
\end{abstract}

Descriptors: Public administration; old age; job; social justice; legal guarantees. (words takenfrom UNESCO Thesaurus). 


\section{INTRODUCCIÓN}

La presente investigación aborda el problema de la discriminación para el ascenso y cese en el trabajo, de las personas adultas mayores en las entidades del servicio público en el Ecuador. Por su parte, el Ministerio de Inclusión Económica y Social (2012) en la agenda de igualdad para adultos mayores señala que el envejecimiento es un proceso multidimensional -que tiene incidencia en la persona, familia y comunidad, implica la puesta en marcha de acciones integrales, solidarias, que contribuya a revalorizar el rol de las personas adultas mayores en la sociedad. Así mismo, la Organización Internacional de Trabajo(1980) en la recomendación 162 establece en el numeral 2 que los problemas de empleo de los trabajadores de edad deberían tratarse en el contexto de una estrategia global y equilibrada de pleno empleo y, a nivel de las empresas, de una política social, global y equilibrada, además; menciona que en el marco de una política nacional destinada a promover la igualdad de oportunidades y de trato para los trabajadores, sea cual fuere su edad, y en el marco de su legislación y práctica relativas a tal política, la OIT además es clara en mencionar que todo miembro debe adoptar medidas para impedir la discriminación respecto de los trabajadores de edad en materia de empleo y de ocupación. El fenómeno del envejecimiento poblacional exige mirar desde nuevas ópticas el papel de las personas mayores y sus contribuciones, no sólo desde lo económico, sino desde lo socio-cultural. (Maldonado Pedroza y Yánez Contreras, 2014, p. 103)

Por tal razón, las personas adultas mayores son titulares de derechos en varios Instrumentos Internacionales, sin contar con que el Estado está en la obligación de velar por el cumplimiento de sus derechos, pero el problema es en la normativa legal del servicio público, puesto se les discrimina por la edad para el ascenso y cese en el puesto de trabajo, sin importar el aporte que puede brindar, tomando en cuenta sus capacidades y limitaciones. Por tanto, el tema de investigación, es de actualidad y novedoso pues la ley vigente discrimina a las personas adultas mayores y el problema propuesto no ha sido objeto de estudio, desde la visión que se ha propuesto.

De igual manera Zambrano Barquín (2014) plantea: 
La exclusión social de las personas adultas mayores del sector público, que como lo establece la Constitución de la República del Ecuador, son un grupo vulnerable de atención prioritaria y especializada, se produce en primera instancia por la limitada capacidad laboral de los mismos ya que al momento de ser excluidos se produce una desventaja social porque se vulnera uno de sus derechos que es la jubilación. (p.8)

En la actualidad, existen diferente garantías y protección para los adultos mayores, como se muestra en la siguiente relación:

\section{Cuadro 1.}

Garantías-Protección.

\begin{tabular}{|c|c|c|c|}
\hline $\begin{array}{l}\text { Constitución de } \\
\text { la República del } \\
\text { Ecuador (2008) }\end{array}$ & $\begin{array}{l}\text { Ley Orgánica de } \\
\text { las Personas } \\
\text { Adultas Mayores } \\
\text { (2019) }\end{array}$ & $\begin{array}{l}\text { Normativa de } \\
\text { Erradicación de } \\
\text { la } \\
\text { Discriminación. } \\
\text { en el Ámbito } \\
\text { Laboral (2017) } \\
\end{array}$ & $\begin{array}{l}\text { Ley Orgánica de } \\
\text { Servicio Público, } \\
\text { LOSEP (2010) }\end{array}$ \\
\hline $\begin{array}{l}\text { Art.37.El Estado } \\
\text { garantizará a las } \\
\text { personas adultas } \\
\text { mayores los } \\
\text { siguientes } \\
\text { derechos: } \\
2 . \text { El trabajo } \\
\text { remunerado, en } \\
\text { función de sus } \\
\text { capacidades, para } \\
\text { lo cual tomará en } \\
\text { cuenta sus } \\
\text { limitaciones. } \\
\text { Art. 38.- El Estado } \\
\text { establecerá } \\
\text { políticas públicas y } \\
\text { programas de } \\
\text { atención a las } \\
\text { personas adultas } \\
\text { mayores, que } \\
\text { tendrán en cuenta } \\
\text { las diferencias } \\
\text { específicas entre }\end{array}$ & $\begin{array}{l}\text { Art.1.-Objeto. El } \\
\text { objeto de esta Ley } \\
\text { es promover, } \\
\text { regular y } \\
\text { garantizar la plena } \\
\text { vigencia, difusión } \\
\text { y ejercicio de los } \\
\text { derechos } \\
\text { específicos de las } \\
\text { personas adultas } \\
\text { mayores, en el } \\
\text { marco del principio } \\
\text { de atención } \\
\text { prioritaria } \\
\text { especializada, y } \\
\text { expresados en la } \\
\text { Constitución de la } \\
\text { República, } \\
\text { instrumentos } \\
\text { internacionales de } \\
\text { derechos y leyes } \\
\text { humanos y leyes } \\
\text { conexas, con } \\
\text { enfogue de }\end{array}$ & $\begin{array}{lr}\text { Art. 1.- OBJETO. - } \\
\text { El presente } \\
\text { acuerdo tiene } \\
\text { comor objeto } \\
\text { establecer } \\
\text { regulaciones que } \\
\text { permitan el acceso } \\
\text { a los procesos de } \\
\text { selección de } \\
\text { personal en } \\
\text { igualdad r de } \\
\text { condiciones, así } \\
\text { como garantizar la } \\
\text { igualdad y no } \\
\text { discriminación en } \\
\text { el ámbito laboral, } \\
\text { estableciendo } \\
\text { mecanismos de } \\
\text { prevención r de } \\
\text { riesgos } \\
\text { psicosociales. }\end{array}$ & $\begin{array}{l}\text { Las servidoras y } \\
\text { servidores de las } \\
\text { instituciones } \\
\text { señaladas en el } \\
\text { artículo } 3 \text { de esta } \\
\text { ley, cumplidos los } \\
\text { sesenta y cinco (65) } \\
\text { años de edad, } \\
\text { habrán llegado al } \\
\text { tope máximo de su } \\
\text { carrera en el } \\
\text { servicio público, } \\
\text { independientemente } \\
\text { del grado en el cual } \\
\text { se encuentren } \\
\text { ubicados, sin que } \\
\text { puedan ascender. }\end{array}$ \\
\hline
\end{tabular}




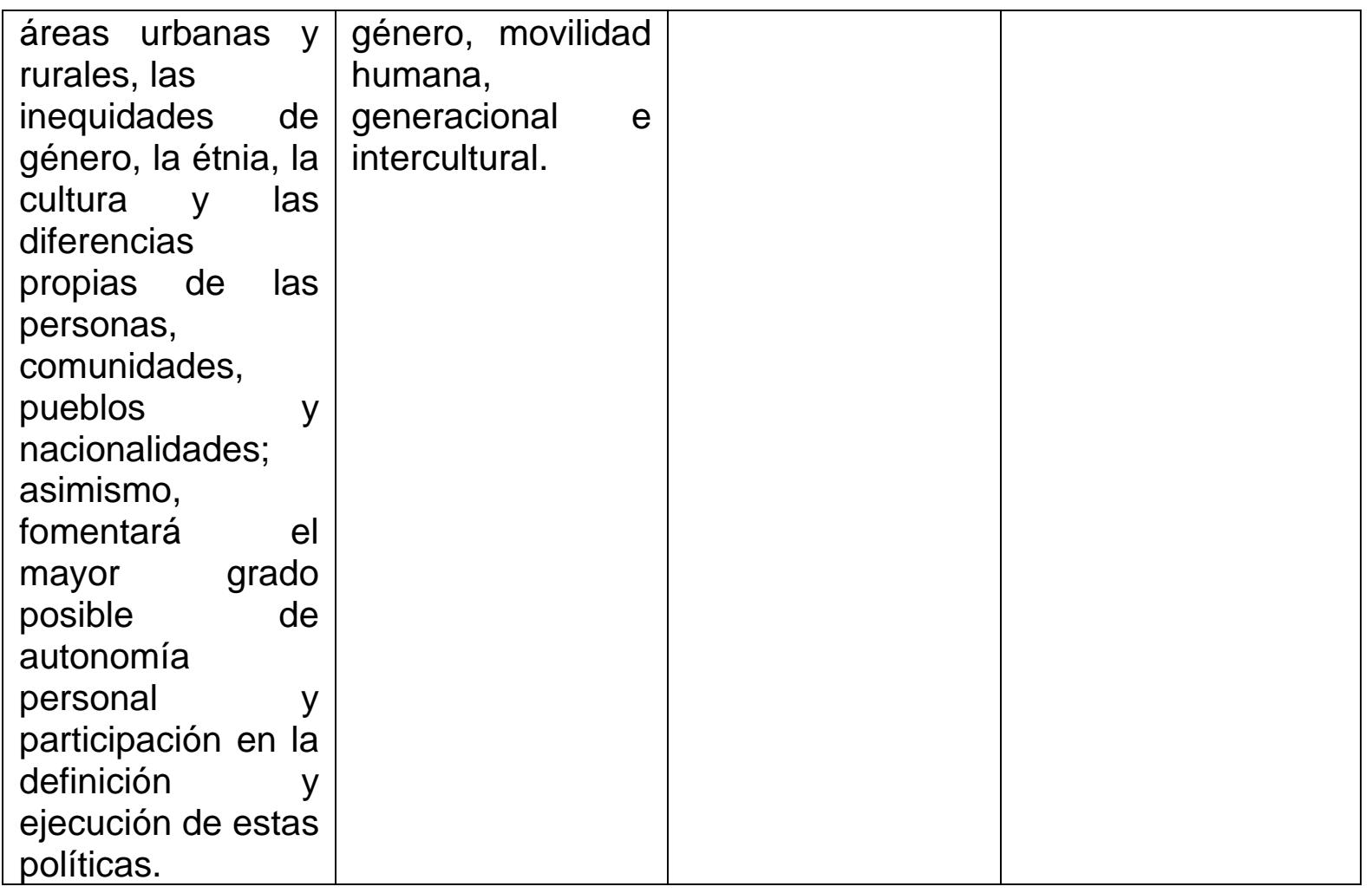

Elaboración: Los autores

En otro aspecto, la falta de empleo en el Ecuador y la necesidad de su impulso, para reactivar la economía; siendo una realidad que la edad se ha convertido en una limitante para conseguir trabajo, ya que los pocos espacios laborales vacantes, permiten mayor posibilidad de acceso a personas entre dieciocho y cuarenta años de edad. Además, el gobierno ha emprendido programas para impulsar el empleo juvenil, que por cierto no está mal, pero se ha olvidado del derecho al trabajo de las personas adultas mayores.

Por tanto, la presente investigación, surge ante la necesidad de reivindicar el derecho constitucional al trabajo dependiente, de las personas adultas mayores que actualmente por mandato legal, se ha restringido en el sector público; por lo que se pretende dar un aporte teórico que permita adecuar el ordenamiento jurídico al estado constitucional de derechos, evitando discriminación a las personas adultas mayores, por su edad. 
Se planteó como objetivo, analizar jurídica y doctrinariamente la discriminación por edad que sufren las personas adultas mayores para el ascenso y permanencia en el servicio público.

\section{METODOLOGÍA}

Dada la naturaleza cualitativa de la investigación, se procedió con el análisis jurídico del ascenso y cese de las personas adultas mayores en el servicio público, en Ecuador, se examinaron el contenido de fondo del análisis documental y bibliográfico, se concibió el uso de métodos de nivel teórico y de nivel empírico del conocimiento, como son: Analítico -sintético, inductivo-deductivo, la información se seleccionó a través de la entrevista y la técnica bibliográfica, apoyándose en la guía de entrevista y las fichas bibliográficas. Para la recolección de datos fácticos acerca de la problemática estudiada, se utilizó la técnica de la entrevista con el propósito de obtener información relevante; esta técnica fue aplicada a tres informantes clave; un profesional del derecho, un Juez del cantón Santo Domingo, y a un Asambleísta de la provincia de Santo Domingo de los Tsáchilas. El estudio jurídico como una estructura compleja, integrada y conformada por la constitución, leyes y códigos, constituidos bajo un subsistema jurídico con características y funciones propias y específicas que interactúan entre sí. (Romero Heras, Erazo Alvares, Pinos Jaén y Narváez Zurita, 2020).

\section{RESULTADOS}

A continuación, se presentan los resultados de las entrevistas realizadas en la presente investigación: 


\section{Cuadro 2.}

Interrogantes-respuestas.

\begin{tabular}{|c|c|}
\hline \multicolumn{2}{|c|}{$\begin{array}{c}\text { Pregunta } 1 \\
\text { ¿Considera Usted que cumplir } 65 \text { años sea un limitante para ascender a puestos de mayor } \\
\text { jerarquía en el sector público? }\end{array}$} \\
\hline Abogado & $\begin{array}{l}\text { Considera que el artículo carece de legalidad jerárquica ya que se } \\
\text { contrapone con lo expresado en la constitución en relación a los } \\
\text { derechos del trabajo para los adultos mayores. }\end{array}$ \\
\hline Juez & $\begin{array}{l}\text { Manifiesta que no es un limitante cumplir } 65 \text { años de edad, ya que } \\
\text { para ejercer un cargo de mayor jerarquía deben seguir } \\
\text { capacitándose. }\end{array}$ \\
\hline Asambleísta & $\begin{array}{l}\text { Sostiene que los servidores públicos al cumplir } 65 \text { años de edad no } \\
\text { están limitados para seguir avanzando en su carrera como servidores } \\
\text { públicos. }\end{array}$ \\
\hline
\end{tabular}

Fuente: Entrevista

\section{Cuadro 3.}

Interrogantes-respuestas.

\begin{tabular}{|c|c|}
\hline \multicolumn{2}{|c|}{$\begin{array}{c}\text { Pregunta } 2 \\
\text { ¿Conoce personas que hayan ascendido a un cargo de mayor jerarquía en el sector público } \\
\text { de más de } 65 \text { años? }\end{array}$} \\
\hline Abogado & $\begin{array}{l}\text { Comentó que ver personas de más de } 65 \text { años de edad en los cargos } \\
\text { del sector público son comunes ya que no solamente se incluyen los } \\
\text { servidores públicos escogidos por pruebas de mérito y oposición, si } \\
\text { no también se incluyen a los elegidos por Votación popular. }\end{array}$ \\
\hline Juez & $\begin{array}{l}\text { Manifiesta que ha conocido personas que se desempeñaron en el } \\
\text { sector público, de } 65 \text { años o más, que han ascendido en la carrera } \\
\text { del servicio público, pese a la limitación que existe en la Ley Orgánica } \\
\text { de Servicio Público. }\end{array}$ \\
\hline Asambleísta & $\begin{array}{l}\text { Contestó que a lo largo de su carrera en el sector público no ha tenido } \\
\text { la oportunidad de conocer a un servidor público que haya cumplido } \\
\text { los } 65 \text { años, fuese ascendido a un cargo de mayor jerarquía debido } \\
\text { a la limitación que pone la Ley Orgánica de Servicio Público en } \\
\text { relación con la edad de una persona. }\end{array}$ \\
\hline
\end{tabular}

Fuente: Entrevista 


\section{Cuadro 4.}

Interrogantes-respuestas.

\begin{tabular}{|c|c|}
\hline \multicolumn{2}{|c|}{$\begin{array}{l}\text { Pregunta } 3 \\
\text { ¿Sabe usted si en la actualidad existe alguna persona de más de } 70 \text { años que se encuentre } \\
\text { trabajando en el sector público? }\end{array}$} \\
\hline Abogado & $\begin{array}{l}\text { Manifiesta en su transcurso en la carrera en el servicio público no ha } \\
\text { conocido persona de más de } 70 \text { años que se encuentre laborando en } \\
\text { alguna institución pública, ya que el Estado, cumpliendo los } 70 \text { años } \\
\text { los obliga a jubilarse en apego al artículo } 81 \text { de la Ley Orgánica de } \\
\text { Servicio Público, LOSEP. }\end{array}$ \\
\hline Juez & $\begin{array}{l}\text { Expresa que no conoce personas de más de } 70 \text { años que trabajen en } \\
\text { el sector público, ya que el Estado obliga al servidor público al } \\
\text { jubilarse, tal como está expresado en el artículo } 81 \text { de la Ley Orgánica } \\
\text { de Servicio Público. }\end{array}$ \\
\hline Asambleísta & $\begin{array}{l}\text { Conforme a su experiencia y trayectoria como servidor público dice } \\
\text { no haber conocido servidor público mayor a } 70 \text { años de edad, ya que } \\
\text { para ellos la jubilación llegada tal edad es obligatoria y opcional desde } \\
\text { los } 65 \text { años de edad, por eso es que muchos compañeros servidores } \\
\text { públicos han optado por la jubilación antes de llegar a la edad máxima } \\
\text { que determina la ley. }\end{array}$ \\
\hline
\end{tabular}

Fuente: Entrevista.

\section{Cuadro 5.}

Interrogantes-respuestas.

\begin{tabular}{|l|l|}
\hline \multicolumn{2}{|c|}{ ¿Cree usted que el Estado garantiza el derecho al trabajo a las personas adultas mayores? } \\
\hline Abogado & $\begin{array}{l}\text { Sostiene que el Estado no garantiza el derecho al trabajo de las personas } \\
\text { mayores, que existen personas que llegando a la edad de } 65 \text { años en el } \\
\text { servicio público, son obligados a firmar renuncias o a jubilarse. }\end{array}$ \\
\hline Juez & $\begin{array}{l}\text { Considera que el Estado garantiza completamente el derecho al trabajo de } \\
\text { las personas adultas mayores ya que la Constitución de la República, } \\
\text { garantizan que el adulto mayor pueda trabajar en función de sus } \\
\text { capacidades y limitaciones. }\end{array}$ \\
\hline Asambleísta & $\begin{array}{l}\text { No cree que el Estado garantice el derecho al trabajo por el mismo motivo } \\
\text { que en la creación de la Ley Orgánica de Servicio Público, se perjudicó } \\
\text { directamente a las personas adultas mayores de edad, no permitiéndoles } \\
\text { continuar ascendiendo en su carrera del servicio público, tal como lo expresa } \\
\text { el artículo 81, evidenciado que el espíritu de esta ley es discriminatorio hacia } \\
\text { los adultos mayores. }\end{array}$ \\
\hline
\end{tabular}

Fuente: Entrevista. 


\section{Cuadro 6.}

Interrogantes-respuestas.

\begin{tabular}{|l|l|}
\hline \multicolumn{2}{|c|}{ ¿Considera usted que es necesaria la experiencia de las personas para el ascenso a un } \\
cargo en el sector público?
\end{tabular}

Fuente: Entrevista.

\section{Cuadro 7.}

Interrogantes-respuestas.

\begin{tabular}{|l|l|}
\hline \multicolumn{2}{|c|}{ ¿Considera usted que el Estado está cumpliendo con su obligación de velar por los } \\
derechos Constitucionales las personas adultas mayores?
\end{tabular}


Fuente: Entrevista.

\section{DISCUSIÓN}

De los resultados obtenidos de la entrevista, se determina que, primero el trabajo en la sociedad es sinónimo de honra, realce y consideración, ya que se garantiza que el trabajador tenga ingresos económicos, se siente parte activa de la sociedad, dándole así una especie de seguridad en el nivel de vida que quiera llevar la persona. El obligar a una persona adulta mayor a jubilarse, acarrea que la actividad económica de este, cese y muchas veces se vea aislado de la sociedad, ya que deja de formar parte de los individuos económicamente activos, causando que dejen de sentirse útiles y capaces, buscando consigo el aislamiento y la soledad, lo cual es un desperdicio para la sociedad, ya que se impide del aprovecho de las capacidades y experiencia que un adulto mayor aún puede brindar.

Es una realidad que la vejez es parte de la vida, a medida que vamos envejeciendo, nuestros cuerpos experimentan cambios que son producto de la edad, pero esto no es ningún impedimento para desempeñar correctamente un cargo que en este caso es público, ya que con la edad es sabido que se obtiene habilidades y virtudes propias de una persona con experiencia. Lamentablemente en Ecuador esto se reduce a una idea utópica, porque los adultos mayores por causa de los prejuicios que tienen acerca de lo que pueden aportar, son rechazado y excluidos de los trabajos o de actividades que se requiera un conocimiento más actualizado en tecnología. Es importante que los adultos mayores sean incluidos y aceptados para que quieran seguir laborando y así la sociedad se retroalimente de la experiencia y sabiduría que por el mero hecho de haber vivido más tiempo, un adulto mayor tiene.

La edad no es un limitante para el ascenso a un puesto de mayor jerarquía y muy por el contrario se debería considerar a los servidores públicos de larga trayectoria, que hayan cumplido los 65 años de edad al beneficio de las instituciones pública en puestos de mayor rango. Así mismo el Articulo 81 de la LOSEP en sus inciso cuarto 
y sexto es contradictorio e incongruente con los preceptos expuestos en párrafos anteriores, ya que tiene como finalidad, limitar el ascenso de un servidor público que cumpla 65 años de edad y forzar la jubilación y el cese del puesto de trabajo al cumplir 70 años de edad.

\section{CONCLUSIONES}

Se considera personas adultas mayores a quienes han cumplido 65 años de edad, (Art $36 \mathrm{CRE}$ ) quienes además forman parte de los grupos de atención prioritaria. Las personas adultas mayores, tienen derecho al trabajo remunerado y el estado es el responsable de procurar programas para la inserción laboral; en Ecuador se prohíbe la discriminación por edad (Art 11 numeral $2 \mathrm{CRE}$ ). La Constitución es la norma suprema y prevalece sobre cualquier otra del ordenamiento jurídico Art. 424 Por lo que ninguna Ley puede limitar derechos reconocidos en la Constitución. Garantizándoles a los servidores públicos mayores de 65 años de edad, seguir ascendiendo en la carrera del servicio público hasta que consideren necesaria una jubilación, de esta forma proporcionándoles una mejor calidad de vida al seguir siendo ente productivo para la sociedad y para sí mismo.

Se propone como solución interponer la garantía jurisdiccional de acción de inconstitucionalidad al artículo 81 de la Ley Orgánica de Servicio Público, la cual puede ser presentada por cualquier persona o grupo de persona, en calidad de funcionario público o por su propia cuenta, que tiene la finalidad de dejar sin efecto un acto normativo que sea contraria a la constitución por forma o fondo tal como expresa el artículo 98 de Ley Orgánica de Garantías Jurisdiccionales y Control Constitucional(2009).

\section{FINANCIAMIENTO}

No monetario. 


\section{AGRADECIMIENTO}

A la Universidad Regional Autónoma de los Andes, Santo Domingo, por motivar el desarrollo de la Investigación.

\section{REFERENCIAS CONSULTADAS}

Asamblea Nacional Constituyente de la República del Ecuador, (2008). Constitución de la República del Ecuador. [Constitution of the Republic of Ecuador]. Montecristi. Registro Oficial 449 de 20-oct-2008. Recuperado de https://n9.cl/sia

Asamblea Nacional (2009) Ley Orgánica de Garantías Jurisdiccionales y Control Constitucional. [Organic Law on Jurisdictional Guarantees and Constitutional Control]. Ley 0 Registro Oficial Suplemento 52 de 22-oct-2009. Oficio No. SAN2009-077. Recuperado de: https://n9.cl/su7v

Asamblea Nacional (2019) Ley Orgánica de las Personas Adultas Mayores. Oficio N. T. 343-SGJ-9-0309. Recuperado de: https://n9.cl/ejfk

Constitución de la República del Ecuador (2017) Normativa Erradicación de la Discriminación en el Ámbito Laboral. [Regulations Eradication of Discrimination in the Workplace Acuerdo Ministerial 82 Registro Oficial 16 de 16-jun.-2017 Estado: Vigente No. MDT-2017-0082. Recuperado de: https://n9.cl/y1rei

Maldonado Pedroza, C. y Yánez Contreras, M. (2014). Una aproximación al estudio del empleo en la tercera edad. [An approach to the study of employment in the elderly]. Cuadernos del Cendes, 31(86), 95-110. Recuperado de: https://n9.cl/ga3vd

Ministerio de Inclusión Económica y Social. (2012). Agenda de igualdad para adultos mayores 2012-2013.[ Equality Agenda for Older Adults 2012-2013]. Recuperado de: https://n9.cl/r8ybc

Organización Internacional de Trabajo(1980). Recomendación sobre los trabajadores de edad.[ Recommendation on older workers].Recuperado de: https://n9.cl/wfs8m

Presidencia de la República (2010) Ley Orgánica de Servicio Público. [ Organic Law on Public Service]. Registro Oficial № 294. Miércoles 6 de octubre del 2010. https://n9.cl/do7v7 
Romero Heras, J., Erazo Álvarez, J, Pinos Jaén, C., y Narváez Zurita, C. (2020). Desnaturalización de acciones afirmativas en el acceso a la educación superior en el Ecuador a favor de las víctimas de violencia de sexo o género. [ Denaturalization of affirmative actions in access to higher education in Ecuador in favor of victims of gender or gender violence]. Iustitia Socialis, 5(8), 50-72. http://dx.doi.org/10.35381/racji.v5i8.561

Zambrano Barquín, M. (2014) La jubilación voluntaria de los servidores públicos y la vulneración de los derechos de las personas adultas mayores. [ The voluntary retirement of public servants and the violation of the rights of older persons]. Trabajo de graduación previo a la obtención del título de Abogada de los Juzgados y Tribunales de la República del Ecuador. Universidad Técnica de Ambato. Recuperado de: https://n9.cl/kb7nz 